\title{
Bit Plane Encoding and Encryption ${ }^{\star}$
}

\author{
Anil Yekkala and C.E. Veni Madhavan \\ ${ }^{1}$ Philips Electronics India Ltd., Bangalore \\ anil.yekkala@philips.com \\ ${ }^{2}$ Indian Institute of Science, Bangalore \\ cevm@csa.iisc.ernet.in
}

\begin{abstract}
The rapid growth in multimedia based Internet systems and applications like video telephony, video on demand and also tele-medicine has created a great need for multimedia security. One of the important requirements for multimedia security is encryption. Owing to the size of multimedia data and real time requirements, lightweight encryption schemes are important. Lightweight encryption schemes are based on taking the structure of multimedia data into consideration and partially encrypting the content. Even though several lightweight encryption schemes are existing for lossy compression schemes, their do not exist any lightweight encryption scheme for lossless compression. In this paper we present a lossless compression scheme for image, and we show how this scheme can be also used for supporting scalable lightweight encryption.
\end{abstract}

\section{Introduction}

The rapid growth in multimedia based Internet systems and applications like video telephony, video on demand, network based DVD recorders and IP television have created a substantial need for multimedia security. One of the important requirements for multimedia security is transmission of the digital multimedia content in a secure manner using encryption for protecting it from eavesdropping. The simplest way of encrypting multimedia content is to consider the two-dimensional/three-dimensional image/video stream as an one-dimensional stream and to encrypt the entire content using standard block ciphers like AES, DES, IDEA or RC4 or using a stream cipher. The method of encrypting the entire multimedia content is considered as a naive encryption approach.

Even though the naive encryption approach provides the desired security requirements, it imposes a large overhead on the multimedia codex. This is due to the size of the multimedia content, and also due to real time requirements of transmission and rendering. Hence, lightweight encryption schemes are gaining popularity for multimedia encryption. Lightweight encryption schemes are based on the principle "encrypt minimal and induce maximum noise". Lightweight encryption schemes are designed to take the structure of the multimedia content into consideration.

\footnotetext{
* This work was done by the author as part of his Msc(Engg) thesis at Indian Institute of Science, Bangalore.
} 
Several lightweight encryption schemes exist for lossy compression [5], [1], [2, 3] and [7], as well as few schemes exist for uncompressed data [6], 4]. But we do not see any schemes for encrypting multimedia data within lossless compression. Even though lossy compression schemes are generally acceptable for most applications. But for some applications like medical imaging any form of loss is not acceptable. In these cases the images and video are generally stored in lossless or uncompressed format. The only approach currently available is to encrypt the content after lossless compression. In current standard lossless compression schemes, the compression factor is generally less then 2. Thus, encrypting the entire compressed content after lossless compression will still prove to be an overhead. In this section we propose a new lossless encoding scheme for images, which permits scalable lightweight encryption scheme.

\section{Proposed Scheme}

The proposed lossless encoding and encryption scheme for image uses two important properties of image data, one for designing the encoding scheme and one for designing the encryption scheme.

Firstly, compression within an image can be achieved by dividing it into bit planes, and using the spatial redundancy within each bit plane. This is due to the fact that the MSB bit planes will have longer runs of 1's or 0's. This fact can be observed from Table 1 . From the table it can be observed except for baboon image, every other image have more than $60 \%$ of runs of length greater then equal to 8 in their $M S B 0$ plane. Similarly for $M S B 1$, more then $45 \%$ of runs are of length greater then equal to 8 . Similarly, the percentage of run lengths greater then equal to 4 have been computed for the remaining bit planes. It is seen from the table that long runs are generally present for bit planes $M S B 0$ to $M S B 4$, and as expected the percentage of long runs decrease from bit plane $M S B 0$ to bit plane $M S B 7$. Hence, an efficient way of encoding the bit planes will be to encode the run's using a variable length encoding scheme.

Secondly, most of the information within an image is present in its most significant bit planes, and hence encrypting the most significant bit-planes is sufficient for purpose of confidentiality. The number of most significant bit planes to be

Table 1. Percentage of long runs in various bit planes

\begin{tabular}{|l|c|c|c|c|c|c|c|}
\hline & \multicolumn{7}{|c|}{ Images } \\
\cline { 2 - 8 } \% of long runs in each bit plane & Baboon & Bandon & Brandyrose & Lena & Opera & Peppers & Pills \\
\hline \% runs of length $\geq 8$ in $M S B 0$ & 34.6 & 80.7 & 86.2 & 66.7 & 62.2 & 75.7 & 81.8 \\
\% runs of length $\geq 8$ in $M S B 1$ & 18.6 & 70.4 & 60.0 & 45.3 & 46.3 & 53.8 & 57.1 \\
\% runs of length $\geq 4$ in $M S B 2$ & 27.3 & 78.4 & 66.2 & 54.8 & 58.2 & 52.6 & 64.9 \\
$\%$ runs of length $\geq 4$ in $M S B 3$ & 17.3 & 64.2 & 45.6 & 34.6 & 35.9 & 32.5 & 48.6 \\
$\%$ runs of length $\geq 4$ in $M S B 4$ & 13.2 & 45.0 & 24.1 & 21.0 & 20.2 & 17.2 & 32.9 \\
$\%$ runs of length $\geq 4$ in $M S B 5$ & 12.4 & 29.7 & 13.7 & 13.6 & 13.1 & 12.6 & 19.5 \\
$\%$ runs of length $\geq 4$ in $M S B 6$ & 12.4 & 25.5 & 12.5 & 12.6 & 12.6 & 12.6 & 13.5 \\
$\%$ runs of length $\geq 4$ in $M S B 7$ & 12.5 & 24.1 & 12.4 & 12.5 & 12.5 & 12.4 & 12.6 \\
\hline
\end{tabular}


Table 2. Amount of energy in the MSB bit planes

\begin{tabular}{|c|c|c|c|c|c|c|c|}
\hline \multirow{2}{*}{ \% of energy } & \multicolumn{7}{|c|}{ Images } \\
\cline { 2 - 7 } & Baboon & Bandon & Brandyrose & Lena & Opera & Peppers & Pills \\
\hline \% energy in MSB 0 & 46.28 & 42.91 & 43.23 & 47.20 & 38.08 & 44.97 & 41.45 \\
\% energy in MSB 0-1 & 60.15 & 62.12 & 68.54 & 65.70 & 58.62 & 62.58 & 68.87 \\
\% energy in MSB 0-2 & 79.55 & 78.53 & 81.97 & 79.87 & 79.12 & 79.56 & 82.19 \\
\% energy in MSB 0-3 & 89.91 & 88.77 & 91.08 & 89.88 & 89.70 & 90.01 & 90.80 \\
\% energy in MSB 0-4 & 95.19 & 94.67 & 95.74 & 95.19 & 95.00 & 95.23 & 95.58 \\
\% energy in MSB 0-5 & 97.92 & 97.65 & 98.15 & 97.91 & 97.83 & 97.94 & 98.08 \\
\% energy in MSB 0-6 & 99.30 & 99.19 & 99.38 & 99.30 & 99.27 & 99.31 & 99.36 \\
\% energy in MSB 0-7 & 100.00 & 100.00 & 100.00 & 100.00 & 100.00 & 100.00 & 100.00 \\
\hline
\end{tabular}

encrypted will depend upon the amount of security required. The amount of the information present in the most significant bit planes can be observed from the Table 2 The table shows the percentage of energy present in the most significant bit planes. From the Table 2 it can be clearly observed that approximately $40 \%-45 \%$ of the energy is present in the $M S B 0$, whereas approximately $80 \%$ of the energy is present in the first 3 most significant bit planes, namely $M S B 0$, $M S B 1$ and $M S B 2$.

Based on the observation that most of the energy within an image is present in the MSB bits planes, a lightweight encryption scheme was proposed by Schmidth et al. in their work [6. Schmidth et al. proposed to divided the entire image into 8 bit planes and encrypt the most significant bit planes. Unfortunately the scheme is limited to images in uncompressed domain and cannot be extended to images compressed in lossless domain. This is due to the fact that the statistics of the image gets disturbed after encryption and the image loses most of its spatial redundancy. Hence the amount of compression that can be gained on using a lossless compression on the encrypted image reduces drastically.

Hence, from the observations on importance of MSB planes for purpose of security and long runs in MSB planes for purpose of compression, an approach can be to divide the image into bit planes and compress each bit plane before applying encryption. Hence a lightweight encryption scheme can be incorporated with a lossless encoding scheme in following manner

1. Divide the image into bit planes. For 8-bit grey image the number of bit planes will be equal to 8 , whereas for color images the number of bit planes will be 24, 8 bit planes for each of the three color code.

2. Encode each bit plane by encoding the run lengths.

3. Encrypt the MSB planes after encoding. The number of MSB planes to be encrypted will depend upon the level of security required.

\subsection{Encoding Algorithm}

Each of the bit planes are scanned row by row starting from the top-left pixel, and the run lengths are computed. For $M S B 0$ and $M S B 1$ bit planes, the maximum 
run length is restricted to 7. The run lengths are encoded using a Huffman coding. The Huffman codewords can be either predefined computed from several training images, or alternatively, it can be computed for the respective image, by computing the relative frequencies of run lengths of size 1-7 and run length of size greater then equal to 8, and subsequently computing the Huffman codeword. The relative frequencies and the corresponding Huffman codewords of run lengths generated from a Lena image for $M S B 0$ and $M S B 1$ bit planes are shown in Table 3.

For $M S B 2$ to $M S B 7$ bit planes, the maximum run length is restricted to 3 . Similar to $M S B 0$ and $M S B 1$ bit planes the Huffman codewords for $M S B 2$ to

Table 3. Frequencies and Huffman codewords for MSB0 and MSB1

\begin{tabular}{|c|c|c|c|c|}
\hline Length of runs & \multicolumn{2}{|c|}{ Frequencies } & \multicolumn{2}{c|}{ Huffman codeword } \\
\cline { 2 - 5 } & MSB0 & MSB1 & MSB0 & MSB1 \\
\hline Runs of length 1 & 0.105 & 0.179 & 011 & 011 \\
Runs of length 2 & 0.064 & 0.114 & 000 & 001 \\
Runs of length 3 & 0.046 & 0.078 & 0100 & 0101 \\
Runs of length 4 & 0.036 & 0.058 & 0011 & 0001 \\
Runs of length 5 & 0.034 & 0.048 & 0010 & 0000 \\
Runs of length 6 & 0.025 & 0.038 & 01011 & 01001 \\
Runs of length 7 & 0.024 & 0.032 & 01010 & 01000 \\
Runs of length $\geq 8$ & 0.667 & 0.453 & 1 & 1 \\
\hline
\end{tabular}

Table 4. Frequencies for MSB2 to MSB7

\begin{tabular}{|c|c|c|c|c|c|c|}
\hline & \multicolumn{5}{|c|}{ Frequencies } \\
\cline { 2 - 7 } Length of runs & MSB2 & MSB3 & MSB4 & MSB5 & MSB6 & MSB7 \\
\hline Runs of length 1 & 0.227 & 0.347 & 0.441 & 0.492 & 0.497 & 0.497 \\
Runs of length 2 & 0.137 & 0.192 & 0.226 & 0.248 & 0.251 & 0.252 \\
Runs of length 3 & 0.088 & 0.115 & 0.122 & 0.124 & 0.126 & 0.125 \\
Runs of length $\geq 4$ & 0.548 & 0.346 & 0.210 & 0.136 & 0.126 & 0.125 \\
\hline
\end{tabular}

Table 5. Compression achieved using bit plane encoding

\begin{tabular}{|c|c|c|c|}
\hline Image & $\begin{array}{c}\text { Original Image Size } \\
\text { (bytes) }\end{array}$ & $\begin{array}{c}\text { Compressed Image Size } \\
\text { (bytes) }\end{array}$ & Compression Ratio \\
\hline Baboon & 262144 & 210643 & 1.244494 \\
Bandon & 245830 & 133377 & 1.843121 \\
Brandyrose & 385392 & 238625 & 1.615053 \\
Lena & 262144 & 176800 & 1.4827 \\
Opera & 407270 & 277194 & 1.469260 \\
Peppers & 262144 & 174890 & 1.498908 \\
Pills & 415200 & 249233 & 1.665911 \\
\hline
\end{tabular}


$M S B 7$ bit planes can either be pre-defined or can be defined from the relative frequencies of run lengths of the respective image. The relative frequencies of various run lengths computed from Lena image for bit planes $M S B 2$ to $M S B 7$ are shown in Table 4

The amount of compression achieved for some standard images using run length encoding on bit planes is shown in Table 5.

\subsection{Encryption Algorithm}

The MSB planes can be encrypted using a standard stream cipher or preferably a standard block cipher in OFB mode. The number of MSB planes to be encrypted can be decided based on the security level required for the underlying content and the system. The scheme in terms of security and computational cost allow eight levels of scalability. At the first level of security, only MSBO plane is encrypted, at the next level i.e. the second level of security $M S B 0$ and $M S B 1$ planes are encrypted. Finally at the eight level of security all the bit planes will be encrypted, which will be equivalent to a naive encryption approach.

\subsection{Extension to Color Images}

For color images the scheme can be extended by following the procedure similar to gray images on all the color components, namely $R$ (red) component, $G$ (green) component and $B$ (blue) component.

\section{Results}

The amount of noise introduced by the encryption scheme at various levels of security measured in terms of PSNR and MAD are shown in Table 6 and Figure 1 From Table 6 it can be observed that the amount of additional noise introduced by encrypting $M S B 2$ to $M S B 7$ planes is negligible. Hence, it can be concluded that encrypting only $M S B 0$ and $M S B 1$ will provide sufficient security (it is to be noted that encrypting only $M S B 0$ was found not to be secured by Schmidth et al. in their work [6]). But, for data with high security requirements it is recommended to encrypt atleast the first four MSB bit planes. The need for encrypting the first four MSB planes can be observed from Figure 2. The figure shows 8 sets of images of Lena. The first image is when all eight bit planes are present; the second image is formed by shifting each eight bit pixel of Lena image left by 1 bit (i.e. image is formed from $M S B 1$ to $M S B 7$ ). Finally the eight image is formed by left shifting each pixel by 7 bits (i.e. image is formed from only $M S B 7$ ). From the figure it can be observed that the original image is visible to certain extent even when only $M S B 4$ to $M S B 7$ are available.

The advantage of compressing the bit planes before encrypting also results in saving of computational time. The computational time saved at various levels of security for Lena image of size 2097152 bits $(=512 \times 512 \times 8)$ by encoding the bit planes before encrypting them is shown in Table 7 First column shows 
Table 6. MAD and PSNR for bit plane encryption scheme

\begin{tabular}{|c|c|c|c|c|c|c|c|c|c|}
\hline \multicolumn{2}{c|}{ Image } & Level1 & Level2 & Level3 & Level4 & Level5 & Level6 & Level7 & Level8 \\
\hline Lena & MAD & 64.04 & 72.25 & 72.30 & 72.91 & 73.03 & 73.06 & 73.07 & 73.07 \\
& PSNR & 8.99 & 8.96 & 9.23 & 9.22 & 9.22 & 9.22 & 9.22 & 9.22 \\
\hline Baboon & MAD & 63.99 & 68.00 & 70.25 & 70.78 & 70.93 & 70.96 & 70.97 & 70.97 \\
& PSNR & 8.99 & 9.58 & 9.54 & 9.53 & 9.53 & 9.53 & 9.53 & 9.53 \\
\hline Peppers & MAD & 64.10 & 71.58 & 74.99 & 75.52 & 75.62 & 75.66 & 75.67 & 75.68 \\
& PSNR & 64.10 & 71.58 & 74.99 & 75.52 & 75.62 & 75.66 & 75.67 & 75.68 \\
\hline Opera & MAD & 64.12 & 71.65 & 72.69 & 73.14 & 73.24 & 73.27 & 73.27 & 73.27 \\
& PSNR & 8.98 & 9.04 & 9.18 & 9.18 & 9.19 & 9.19 & 9.19 & 9.19 \\
\hline Bandon & MAD & 64.04 & 79.25 & 86.12 & 88.98 & 90.31 & 90.87 & 91.13 & 91.25 \\
& PSNR & 8.99 & 8.10 & 7.60 & 7.38 & 7.27 & 7.22 & 7.20 & 7.19 \\
\hline Brandyrose & MAD & 64.04 & 73.57 & 75.02 & 75.36 & 75.47 & 75.50 & 75.51 & 75.51 \\
& PSNR & 8.99 & 8.79 & 8.86 & 8.88 & 8.88 & 8.88 & 8.88 & 8.88 \\
\hline Pills & MAD & 64.13 & 76.07 & 77.83 & 78.42 & 78.55 & 78.58 & 78.59 & 78.59 \\
& PSNR & 8.98 & 8.48 & 8.50 & 8.49 & 8.49 & 8.49 & 8.49 & 8.49 \\
\hline
\end{tabular}

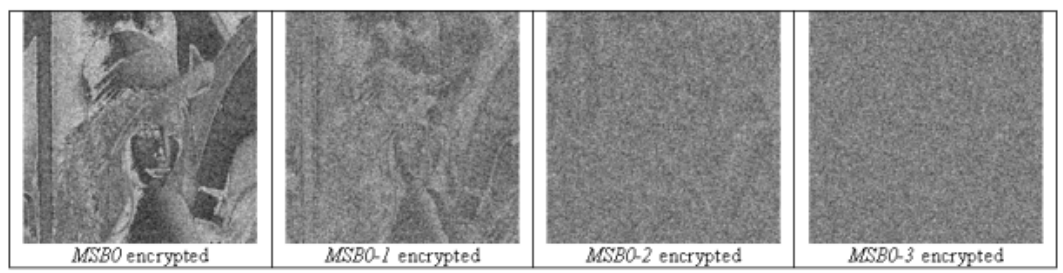

Fig. 1. Lena image encrypted using Bit plane encryption at various levels

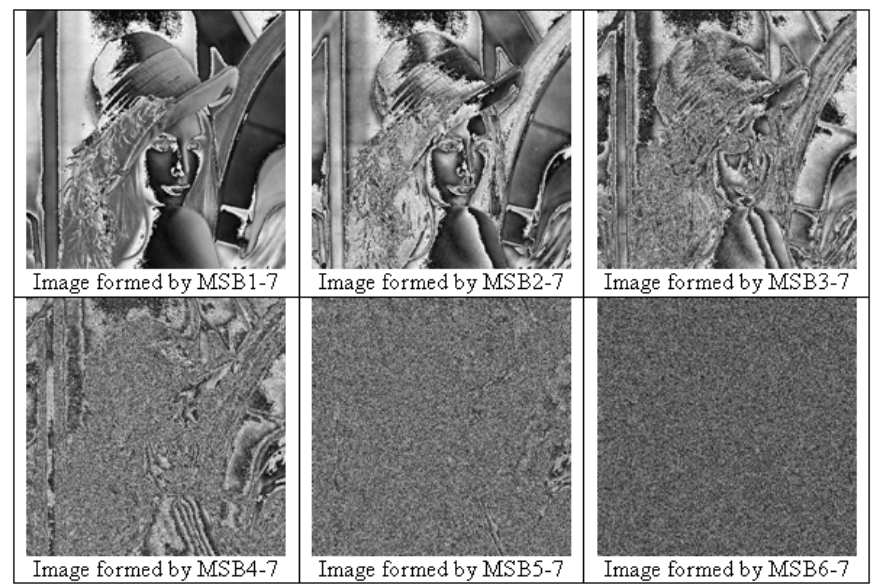

Fig. 2. Lena image formed from its LSBs 
Table 7. Bit plane encryption: gain in computation time due to encoding

\begin{tabular}{|c|c|c|}
\hline Security Level & $\begin{array}{c}\text { \% of total bits encrypted } \\
\text { without compression }\end{array}$ & $\begin{array}{c}\text { \% of total bits encrypted } \\
\text { with bit plane compression }\end{array}$ \\
\hline Level 1 & $12.5 \%$ & $3.4 \%$ \\
Level 2 & $25.0 \%$ & $8.90 \%$ \\
Level 3 & $37.5 \%$ & $15.23 \%$ \\
Level 4 & $50.0 \%$ & $24.30 \%$ \\
Level 5 & $62.5 \%$ & $34.72 \%$ \\
Level 6 & $75.0 \%$ & $45.56 \%$ \\
Level 7 & $90.0 \%$ & $56.50 \%$ \\
Level 8 & $100.0 \%$ & $67.44 \%$ \\
\hline
\end{tabular}

the various security levels i.e from Level-1 to Level-8. Second column shows the percentage of bits that needs to be encrypted at a particular security level compared to encrypting the full uncompressed image when compression is not applied on bit planes. Finally, the third column shows percentage of bits that need to be encrypted at a particular security level using bit plane compression compared to encrypting the full uncompressed image. From the Table 7 it can be observed that using the proposed method only $24.30 \%$ of original uncompressed image needs to be encrypted for encrypting the first four $M S B$ planes. Also from previous discussions it can be concluded that encrypting first four $M S B$ planes provides very high level of security, since very minimal information is present in the first four LSB's.

\section{Conclusions}

In this paper we presented an lossless encoding scheme for images by dividing the image into bit planes and encoding each bit plane individually. The encoding scheme is designed in such a way that it can support partial encryption. The encryption scheme in addition to being secure also supports scalability at eight different levels. A very high level of security can be achieved by encrypting only first four MSB planes, which is equivalent to encrypting roughly only $24 \%$ of bits compared to encrypting the entire image. Moreover since the encryption is achieved by encrypting only the MSB planes, and the MSB planes can be compressed to higher extent compared to the LSB planes, the amount of data to be encrypted is very minimal. The compression achieved may be improved further by using an alternative variable length encoding scheme for encoding the runs of each bit plane. The scheme can be also easily extended for supporting lossless video coding and scalable encryption, by considering each frame of the video as an image. In addition the scheme can be also extended to support progressive encoding, since it can support encoding/encryption and decoding/decryption of the image and video in bit planes starting from MSB's. 


\section{References}

1. Agi, I., Gong, L.: An Empirical Study of Secure MPEG Video Transmission. In: Proceedings of the Symposium on Network and Distributed Systems Security, IEEE, Los Alamitos (1996)

2. Aly, S.: Multimedia Security: Survey and Analysis, Multimedia and Networking Research Lab. CTI, DePaul University, Chicago, http://www.mnlab.cs.depaul.edu

3. Bhargava, B., Shi, C., Wang, Y.: MPEG Video Encryption Algorithms (August 2002), http://raidlab.cs.purdue.edu/papers/mm.ps

4. Choo, E., Lee, J., Lee, H., Nam, G.: SRMT: A Lightweight Encryption Scheme for Secure Real-time Multimedia Transmission. In: International Conference on Multimedia and Ubiquitous Engineering (MUE 2007) (2007)

5. Furht, B., Socek, D., Eskicioglu, A.M.: Chapter 3: Fundamentals of Multimedia Encryption Techniques. In: Multimedia Security Handbook, Published by CRC press LLC(December 2004)

6. Podesser, M., Schmidt, H.P., Uhl, A.: Selective Bitplane Encryption for Secure Transmission of Image Data in Mobile Environments. In: 5th Nordic Signal Processing Symposium on board, Hurtigruten, Norway (October 4-7, 2002)

7. Yekkala, A.K., Udupa, N., Bussa, N., Veni Madhavan, C.E.: Lightweight Encryption for Images. In: IEEE international Conference on Consumer Electronics (2007) 